\title{
Effects of Parasitic Parameters on EMI Filter Performance
}

\author{
Shuo Wang, Student Member, IEEE, Fred. C. Lee, Fellow, IEEE, Dan. Y. Chen, Fellow, IEEE, and \\ Willem Gerhardus Odendaal, Member, IEEE
}

\begin{abstract}
Two filters with identical topologies and components can exhibit a significant difference in filter effectiveness when the layouts are different. The goal of this paper is to examine the effects of parasitic couplings among the filter components and the coupling between the filter components and the ground plane of printed circuit board (PCB). Specifically, six different coupling effects are investigated: the couplings between the inductor and capacitors, a filter inductor and trace loops, two filter inductors, two capacitor parasitic inductances, a filter inductor and ground plane, and two trace loops. Experiments were performed, theories were developed to investigate and characterize these parasitic couplings.
\end{abstract}

Index Terms-Capacitive coupling, EMI filter, inductive coupling, parasitic coupling, transfer gain.

\section{INTRODUCTION}

$\mathbf{I}$ T IS A well-known fact that parasitic elements in the electromagnetic interference (EMI) filter can play an important role in filter performance. Most engineers are well aware of the self-resonant effects of the parasitic winding capacitance of an inductor and the parasitic inductance of a capacitor on filter performance. These effects, while annoying, are still relatively understandable and manageable because they can be measured with an impedance analyzer. The other category of parasitic effects, those related to layout and packaging, are more difficult to decipher. Although this problem is distributed in nature and can theoretically be overcome by solving field problems, this approach is impractical. In the investigation leading to this paper, attempts were made to understand layout-related EMI filter problems. It will be shown that two filters with identical topologies and components can exhibit a significant difference in filter effectiveness when their layouts are different. The investigation will be done from the circuit standpoint so that the resultant conclusions are applicable to many practical problems. Specifically, the impacts of six coupling effects on filter performances are considered. They include the inductive couplings between the following: inductors and printed circuit board (PCB) layout, inductors and capacitors, two capacitors, ground plane and inductors, in and out trace loops and two in-

Manuscript received July 17, 2003; revised November 19, 2003. Recommended by Associate Editor F. Blaabjerg.

S. Wang, F. C. Lee, and W. G. Odendaal are with the Bradley Department of Electrical and Computer Engineering, Virginia Polytechnic Institute and State University, Blacksburg, VA 24061 USA (e-mail: shuowang@ieee.org).

D. Y. Chen was with the Bradley Department of Electrical and Computer Engineering, Virginia Polytechnic Institute and State University, Blacksburg, VA 24061 USA and is now with the Electrical Engineering Department, National Taiwan University, Taipei, Taiwan, R.O.C.

Digital Object Identifier 10.1109/TPEL.2004.826527

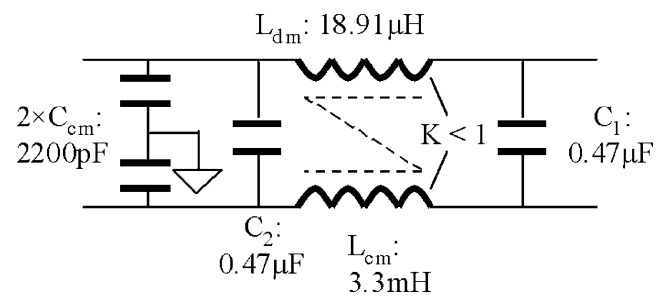

Fig. 1. Circuit of the investigated filter.

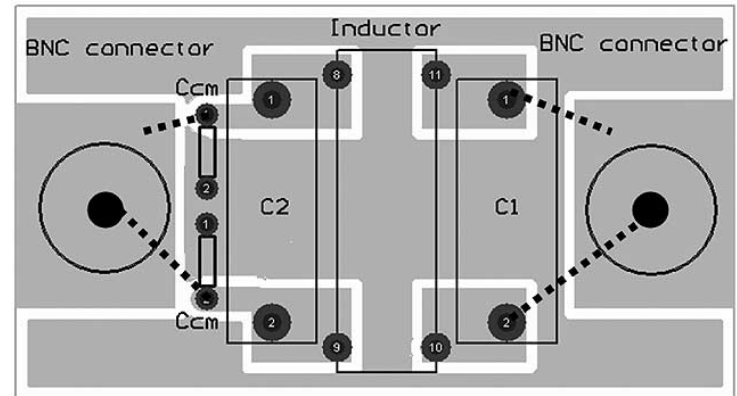

(a)

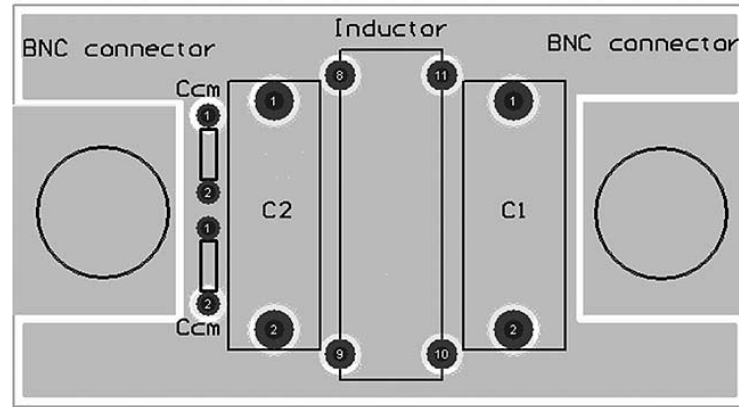

(b)

Fig. 2. PCB layout of the filter: (a) bottom side and (b) top side.

ductors. The effects of these mutual couplings on filter attenuation are identified and quantified in this paper.

\section{PARAsitic Couplings EXISTING IN AN EMI FILTER}

Fig. 1 shows a commonly used EMI filter for AC/DC converters. The PCB layout is shown in Fig. 2, where $C_{1}$ and $C_{2}$ are differential-mode (DM) capacitors, $C_{\mathrm{cm}}$ is common-mode (CM) capacitor which is not investigated here. A CM inductor $L_{\mathrm{cm}}$ is used in the filter. The leakage inductance of $L_{\mathrm{cm}}$, which extends to the air and couples other components, is usually used as DM inductance $L_{\mathrm{dm}}$. Two BNC connectors are attached to the filter for measurement. Fig. 3 shows the equivalent circuit for the DM noise of the circuit in Fig. 1. Also indicated in Fig. 3 


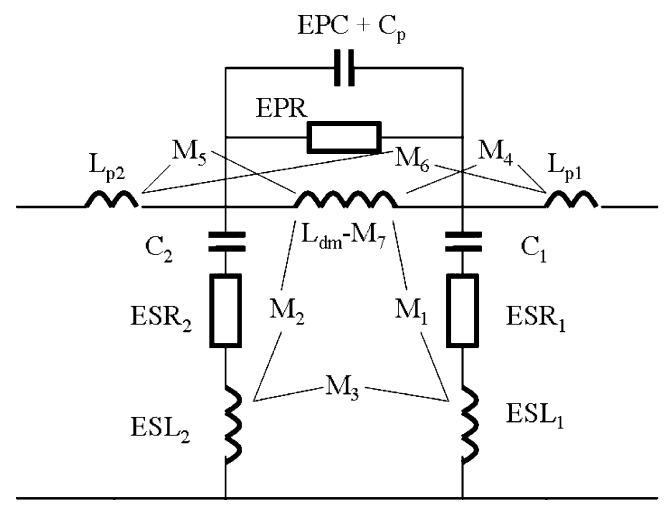

Fig. 3. DM filter model. ( $L_{p 1}, L_{p 2}$ : trace-loop inductance; ESL, ESR, C: capacitor model parameters; $L_{\mathrm{dm}}$, EPC, EPR: DM inductor model parameters; $M_{1}, M_{2}$ : mutual inductance between $L_{\mathrm{dm}}$ and capacitor branches; $M_{3}$ : mutual inductance between two capacitor branches; $M_{4}, M_{5}$ : mutual inductance between $L_{\mathrm{dm}}$ and trace loops; $M_{6}$ : mutual inductance between in and out trace loops; $M_{7}$ : equivalent mutual inductance between ground plane and $L_{\mathrm{dm}} ; C_{p}$ : mutual capacitance between in and out traces).

are eight mutual couplings $M_{1}$ through $M_{7}$ and $C_{p}$. The definitions of these mutual couplings are given in Fig. 3 .

Two kinds of parasitic couplings exist in the filter: inductive coupling and capacitive coupling. Inductive couplings tend to amplify their effects on the branch that has smaller current between two branches with a large current difference. So the inductive couplings, such as those between capacitor branches and the inductor branch, between two capacitor branches, between in and out trace loops, and between inductor and in and out trace loops are the critical parasitic couplings. At the same time, the capacitive couplings tend to amplify their effects on the junction that has lower potential between two junctions with a large potential difference. So the capacitive coupling between in and out traces is a critical coupling for the performance of EMI filters.

In Fig. 3, $M_{1}-M_{7}$ are inductive couplings. $M_{1}, M_{2}$, and $M_{3}$ affect capacitor branches directly. $M_{4}$ and $M_{5}$ affect capacitor branches through branch currents. $M_{6}$ is the coupling between in and out trace loops. $M_{7}$ is the equivalent inductance reduction that occurs due to magnetic flux cancellation between DM inductor and the eddy current in the ground plane. $C_{p}$ is the capacitive coupling between in and out traces. The capacitive coupling happens both directly and through the ground plane between two traces. This capacitive coupling is equivalent to that of a capacitor $C_{p}$ in parallel with the inductor.

\section{IDENTIFYING, ANALYZING AND EXTRACTING PARASITIC COUPLINGS}

\section{A. Inductive Couplings Existing Between DM Inductor $L_{\mathrm{dm}}$ and Capacitor Branches, Between DM Inductor $L_{\mathrm{dm}}$ and In and Out Trace Loops $\left(M_{1}, M_{2}\right.$ and $M_{4}, M_{5}$ in Fig. 3)}

These couplings have similar effects on the capacitor branches. The equivalent circuits for the couplings between inductor and one of the in and out trace loops are shown in Fig. 4. For another trace loop, the equivalent circuit is same. The equivalent circuits for the couplings between inductor and one of the capacitor branches are shown in Fig. 5. For another capacitor branch, the equivalent circuit is also same.

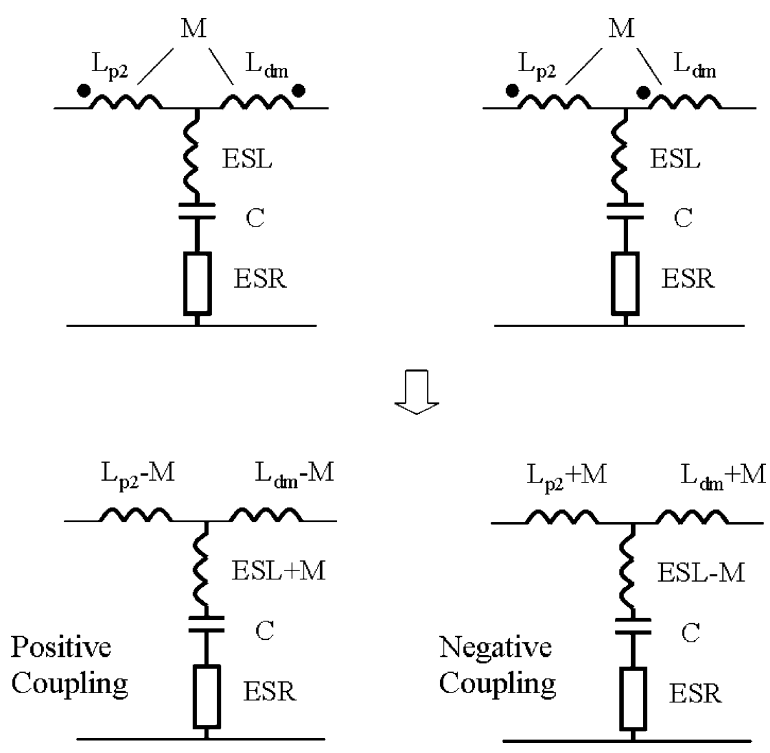

Fig. 4. Inductive coupling between the inductor and the trace loop.
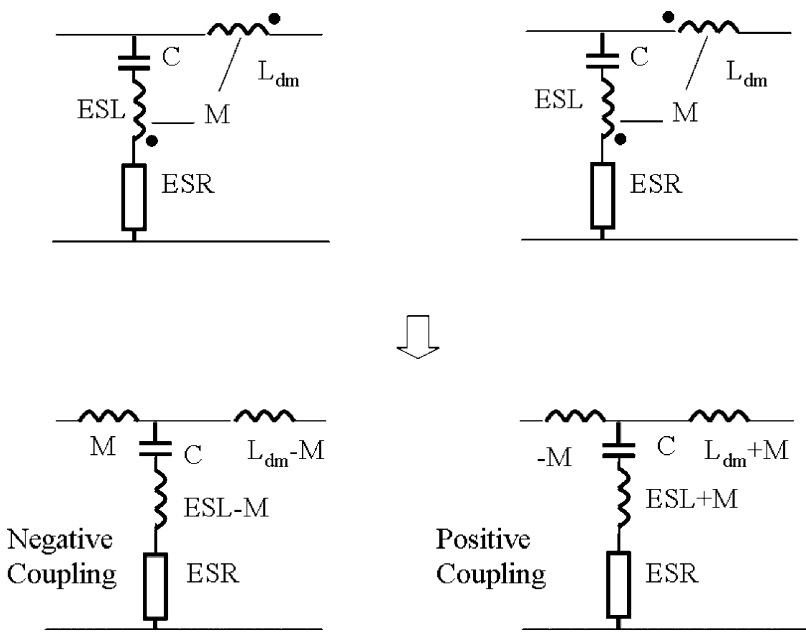

Fig. 5. Inductive coupling between the inductor and the capacitor branch.

Based on the equivalent coupling polarities on the capacitor branch, the positive and negative couplings are defined in Figs. 4 and 5. Considering the different coupling polarities, the two circuits on the top are equivalent to the two circuits on the bottom respectively. In the circuits on the bottom, $M$ is the equivalent inductance on the capacitor branches caused by the couplings of the inductor. If the equivalent inductance on the capacitor branch is positive, the coupling is positive coupling; otherwise it is negative coupling. If these two kinds of couplings exist at the same time, the total effect determines the coupling polarity.

The capacitor branch impedance of the positive coupling is

$$
Z_{1}=\mathrm{ESR}+j\left[\omega(\mathrm{ESL}+M)-\frac{1}{\omega C}\right]
$$

The resonant frequency $f_{1}$ of this capacitor branch is therefore lower than that of the capacitor as

$$
f_{1}=\frac{1}{2 \pi \sqrt{(\mathrm{ESL}+M) C}} .
$$




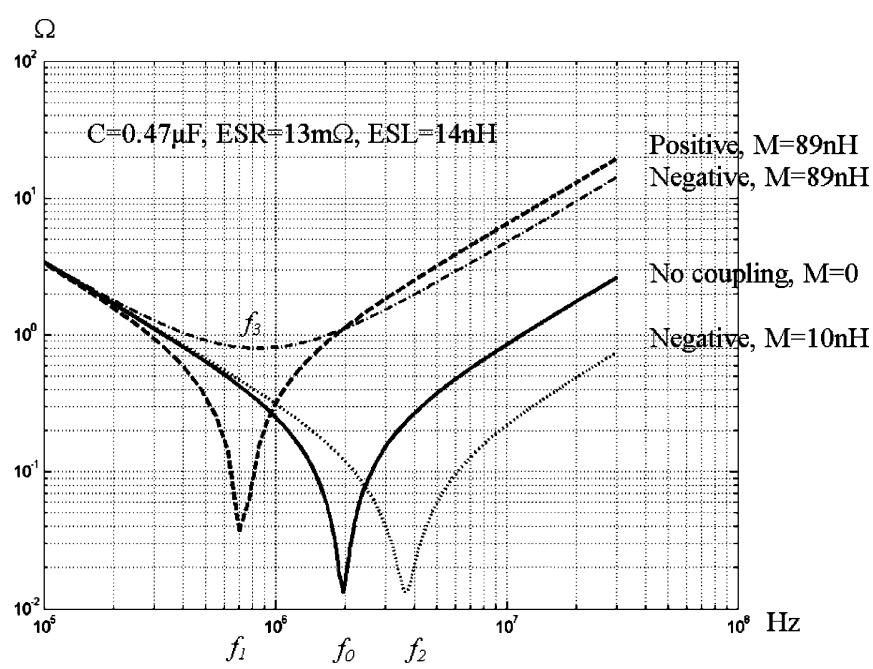

Fig. 6. Calculated impedances for a capacitor branch with different mutual inductances.

The capacitor branch impedance of the negative coupling is

$$
Z_{2}=\mathrm{ESR}+j\left[\omega(\mathrm{ESL}-M)-\frac{1}{\omega C}\right] .
$$

If equivalent series inductance (ESL) of the capacitor is larger than mutual inductance $M$, then the resonant frequency $f_{2}$ of this capacitor branch is higher than that of the capacitor, such that

$$
f_{2}=\frac{1}{2 \pi \sqrt{(\mathrm{ESL}-M) C}} .
$$

In the case when the ESL is smaller than $M$, the minimum impedance instead of resonance happens at frequency $f_{3}$; thus

$$
f_{3}=\frac{1}{2 \pi \sqrt{(M-\mathrm{ESL}) C}} .
$$

The $f_{3}$ is different from $f_{1}$ and $f_{2}$ because no phase polarity change occurred at $f_{3}$. On the contrary, $f_{1}$ and $f_{2}$ are resonant frequencies with a phase polarity change.

And for this case, (3) can be rewritten as

$$
Z_{2}=\mathrm{ESR}+\frac{1}{j \omega\left[\frac{C}{1+\omega^{2} C(M-\mathrm{ESL})}\right]}=\mathrm{ESR}+\frac{1}{j \omega C_{e q}} .
$$

From (6), the capacitor branch can be equivalent to a capacitor $C_{e q}$ whose capacitance is the function of frequencies. After $f_{3}$, as the frequency increases, $C_{e q}$ becomes smaller.

After $f_{1}, f_{2}$ or $f_{3}$, the impedance of the capacitor branch becomes higher as frequency increases. Because the small impedance of the capacitor branch benefits the filter performance, the filter performance would become worse after $f_{1}, f_{2}$ or $f_{3}$. Any couplings affecting the impedance of the capacitor branch would affect the performance of the filter. When the frequency is much higher than the series resonant frequency of the capacitor branch, the impedances are

$$
\left|Z_{1}\right|=|\omega(\mathrm{ESL}+M)| \text {, and }\left|Z_{2}\right|=|\omega(\mathrm{ESL}-M)| \text {. }
$$

From (7), $\left|Z_{2}\right|$ is smaller than $\left|Z_{1}\right|$. If $M$ is equal to ESL, $\left|Z_{2}\right|$ is zero and filter has the best performance in the high frequency (HF) range, while if $M$ is much larger than ESL, $\left|Z_{1}\right|$ is almost

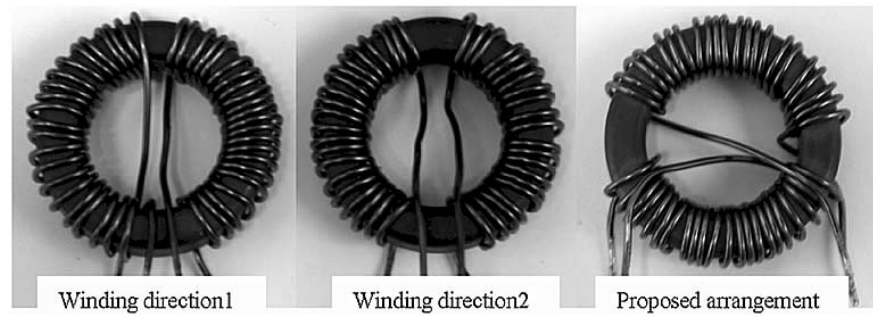

Fig. 7. Different winding structures have different coupling polarities.

TABLE I

EXTRACTED MUTUAL INDUCTANCES

\begin{tabular}{c|c|c}
\hline Mutual inductance & $\begin{array}{c}\text { Between the inductor } \\
\text { and in/out trace loops }\end{array}$ & $\begin{array}{c}\text { Between the inductor } \\
\text { and capacitor branches }\end{array}$ \\
\hline $\begin{array}{l}\text { Winding direction1 } \\
\text { (negative coupling) }\end{array}$ & $18.7 \mathrm{nH}$ & $89.3 \mathrm{nH}$ \\
\hline $\begin{array}{l}\text { Winding direction2 } \\
\text { (positive coupling) }\end{array}$ & $10.3 \mathrm{nH}$ & $83.3 \mathrm{nH}$ \\
\hline Proposed arrangement & $1.8 \mathrm{nH}$ & $7.5 \mathrm{nH}$ \\
\hline
\end{tabular}

equal to $\left|Z_{2}\right|$ and filters have almost the same performance in the $\mathrm{HF}$ range.

The analysis is demonstrated in Fig. 6. In Fig. 6, four computed impedance curves for a capacitor branch are shown. These four cases are: positive coupling, zero coupling and two negative couplings. The capacitance is $0.47 \mu \mathrm{F}$. ESL is $14 \mathrm{nH}$ and equivalent series resistance (ESR) is $13 \mathrm{~m} \Omega$. The mutual inductance is $89 \mathrm{nH}$ for positive coupling, $89 \mathrm{nH}$, and $10 \mathrm{nH}$ for two negative couplings.

In fact, winding directions and arrangement greatly affect the couplings. The distribution of leakage magnetic flux (DM magnetic flux) of a CM inductor is described in [2]. In Fig. 7, winding direction 1 and winding direction 2 have opposite magnetic flux directions. Therefore, the coupling polarities between these two inductors and capacitors are opposite. So are the couplings between the inductors and the trace loops. For the proposed winding arrangement in Fig. 7, two windings are proposed to be rotated by $90^{\circ}$, and they are symmetrical to the capacitors and trace loops. Because the net magnetic flux passing through the capacitors and trace loops is greatly reduced, the couplings are much smaller than those in the previous two winding structures. In the experiments, the self-parasitic parameters of the capacitor, the inductor and the trace loops are extracted using a precision impedance analyzer Agilent $4294 \mathrm{~A}$. The mutual inductances are extracted through scattering parameters (S-parameters) using a network analyzer HP 4195 A. The extracted mutual inductances are shown in Table I.

The equivalent circuits for the left half filter including the self and mutual inductances are shown in Fig. 8 (the filter is symmetrical so the equivalent circuits for the right half is same as that of the left half). The total effects on the capacitor branch are determined by the sum of two kinds of couplings on the capacitor branch. For the investigated EMI filter, when winding direction 1 is used, the equivalent inductance on the capacitor branch caused by the coupling between the inductor and the capacitor branch is negative, which is opposite to that caused by the coupling between the inductor and the trace loop. Because 


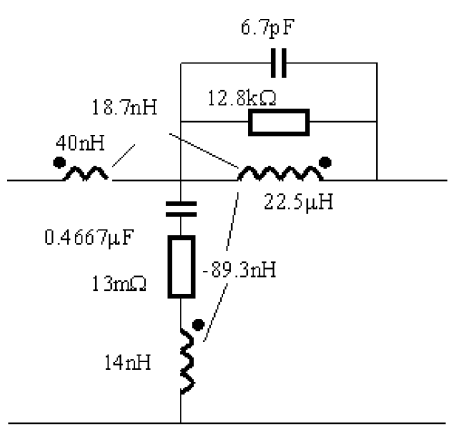

Negative coupling (direction 1)

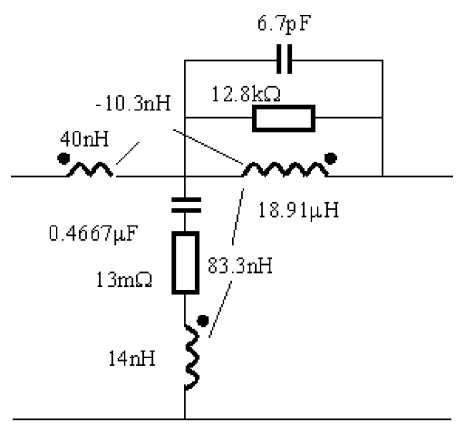

Positive coupling (direction 2)

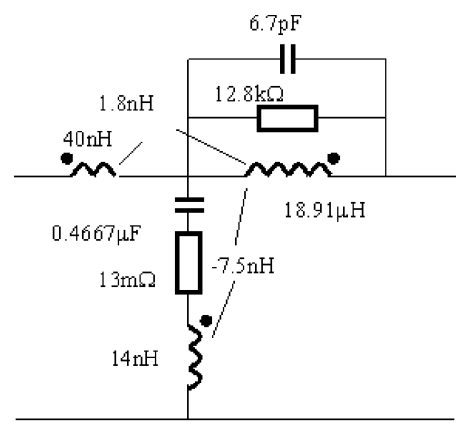

Smaller coupling (Proposed)

Fig. 8. Equivalent circuits including self and mutual inductances.

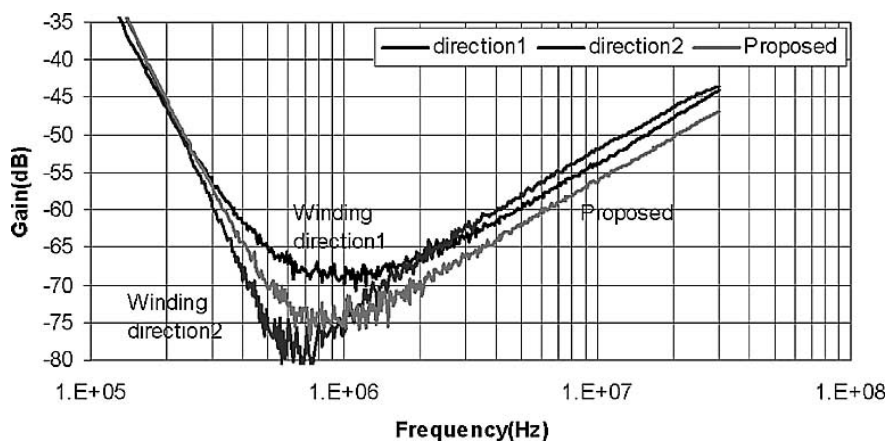

Fig. 9. Measured transfer gains.

the former is larger than the latter, the total equivalent inductance on the capacitor branch is negative. For the winding direction 2 and the proposed winding arrangement, the same principle applies.

The measured transfer gains ( $S_{21}$ of S-parameters) of the whole filter are compared in Fig. 9. The frequency range is from $150 \mathrm{kHz}$ to $30 \mathrm{MHz}$ (EMI standard: EN55022 class B for ac/dc converters). From Table I and Figs. 8 and 9 the transfer gain with positive mutual inductance has the best performance in the low frequency (LF) range, because the series resonant frequency of the capacitor branch is reduced. The transfer gain with negative mutual inductance is only slightly better than the positive case in the HF range because the mutual inductance is much larger than the ESL, just as predicted by (7). The proposed winding arrangement offers good performance in the entire frequency range because the couplings are reduced.

\section{B. Inductive Couplings Existing Between Capacitor Branches ( $M_{3}$ in Fig. 3)}

The inductive coupling between two capacitor branches is generally neglected; however, because there is only one inductor between them on the PCB, the two capacitors are usually close to each other. Because of the large current difference on these two branches, the inductive coupling between them plays a very important role in filter performance. The typical circuit for this effect is shown in Fig. 10. In Fig. 10, the voltage on ESL 1 is

$\dot{U}_{1}=j \omega \dot{I}_{1} \bullet \mathrm{ESL}_{1}+j \omega \dot{I}_{2} \bullet M_{3}=j \omega \dot{I}_{1}\left(\mathrm{ESL}_{1}+\frac{\dot{I}_{2}}{\dot{I}_{1}} M_{3}\right)$.

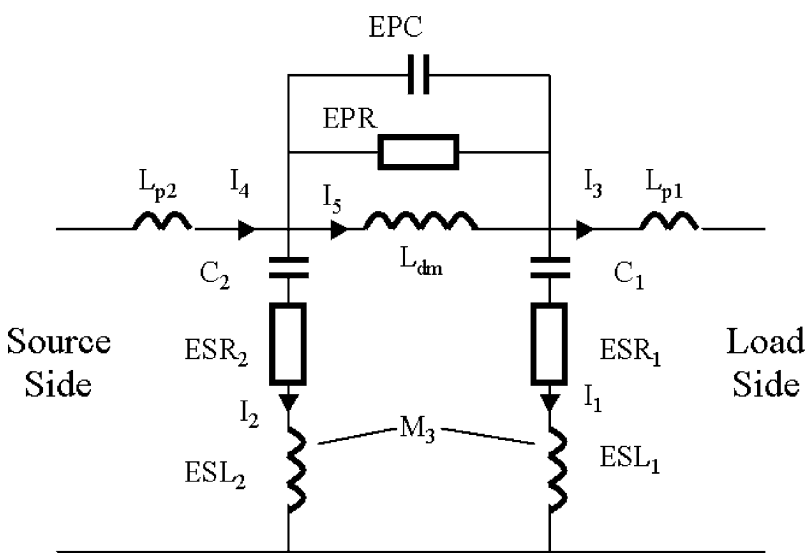

Fig. 10. Equivalent circuit for the coupling between two capacitor branches.

The $I_{1}$ is usually very small compared with $I_{2}$ because most of current is bypassed by the $C_{2}$ branch $\left(I_{2} / I_{1}\right.$ is $40 \mathrm{~dB} / \mathrm{dec}$ after its corner frequency). When the following condition is satisfied:

$$
\frac{I_{2}}{I_{1}}>\frac{\mathrm{ESL}_{1}}{M_{3}}
$$

the mutual inductance would have a much greater effect on the capacitor branch, and thus the filter performance, than the ESL would. When $I_{2} / I_{1}$ is 1,000 , a coupling coefficient of $0.1 \%$ between the two capacitor branches would have obvious effects on the filter performance. In order to demonstrate this effect, experiments are carried out. In the experiment, in order to prevent the influence of other couplings, the in and out trace loops are kept very small and the inductor is mounted on the bottom side of the PCB. The longer distance and two-layer ground plane as a shield can keep the coupling between the inductor and two capacitor branches very small. Three cases are evaluated in the experiment. In the first case, the filter is measured without any actions taken; in the second case, the two capacitors are shielded with 3 mil nickel $\left(\mu_{r}=600\right)$; and in the third case, the two capacitors are shielded with 3 mil nickel and a magnetic shield is placed between them. The experiments are shown in Fig. 11 and the experimental results are shown in Fig. 12.

As shown in Fig. 12, after the capacitors are shielded with 3-mil nickel, there is a 6-dB improvement from $1 \mathrm{MHz}$ to $30 \mathrm{MHz}$. After a magnetic plate was placed between the two capacitors, the improvement increased by $16 \mathrm{~dB}$. It is obvious that the inductive coupling between the two capacitor branches has a significant impact on filter performance. 


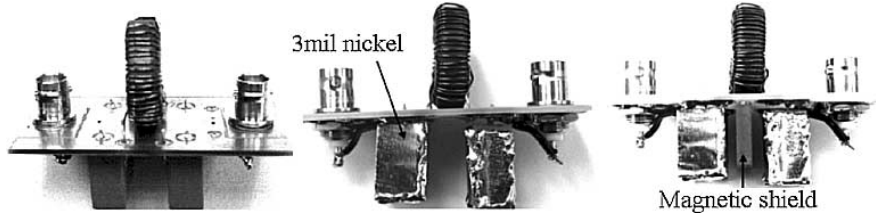

Fig. 11. Three cases in the experiment.

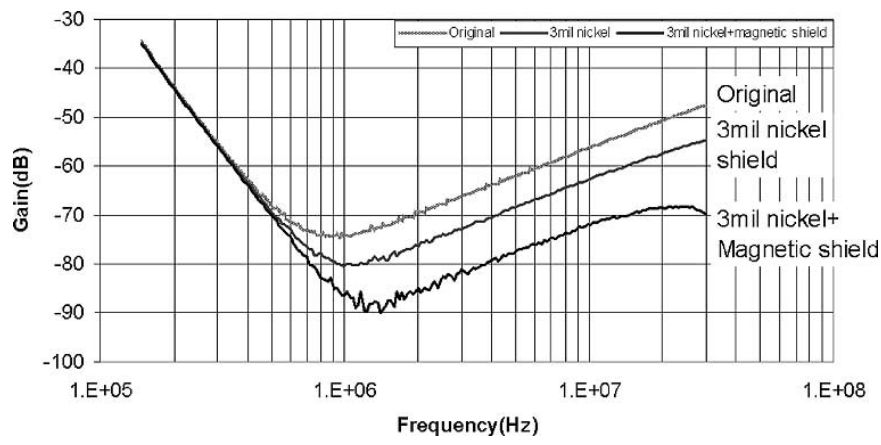

Fig. 12. Comparison of transfer gains.

The mutual inductance between the two capacitor branches is extracted using S-parameters, and the measurement is carried out by a HP 4195 A network analyzer. The extracted mutual inductance between the two capacitor branches is $0.45 \mathrm{nH}$. The corresponding coupling coefficient is $3 \%$. That means a $30 \mathrm{~dB}$ difference between currents on two capacitor branches would have significant effects.

\section{Capacitive Coupling and the Effects of Ground Plane $\left(M_{7}\right.$ and $C_{P}$ in Fig. 3)}

In Fig. 13(a), $C_{p 1}$ and $C_{p 2}$ are the parasitic capacitance between the in and out traces. They are composed of two parts. One part is the direct capacitive couplings between traces: C3 in Fig. 13(b). Another part is capacitive couplings through the ground plane: C1, C2, C4 and C5 in Fig. 13(b). For capacitive couplings through the ground plane, each trace has parasitic capacitors with the ground plane; so the parasitic capacitors between in and out traces are effectively in series through the ground plane. This series capacitance can be larger than that resulting from direct coupling because of the smaller distance between the ground plane and the traces. In Fig. 13(b), the ground plane on the topside of the PCB actually offers a shortcut for the HF noise. The HF noise can reach the output through the parasitic capacitors between the traces and the ground planes instead of coming through the inductor. This effect is modeled by a capacitor $C_{p}$ in parallel with the inductor. Another effect of the ground plane is that it reduces the inductance of the DM inductor because of the eddy current induced in the ground plane. The flux of the DM inductor would induce the eddy current in the ground plane. On the other hand, the opposite flux generated by the eddy current would cancel part of the flux of the DM inductor. As a result, the net flux is reduced, and the DM inductance is thus decreased.

Experiments are carried out using an Agilent 4294 A precision impedance analyzer for an inductor. In the first case, the inductor is measured separately; in the second case the inductor is

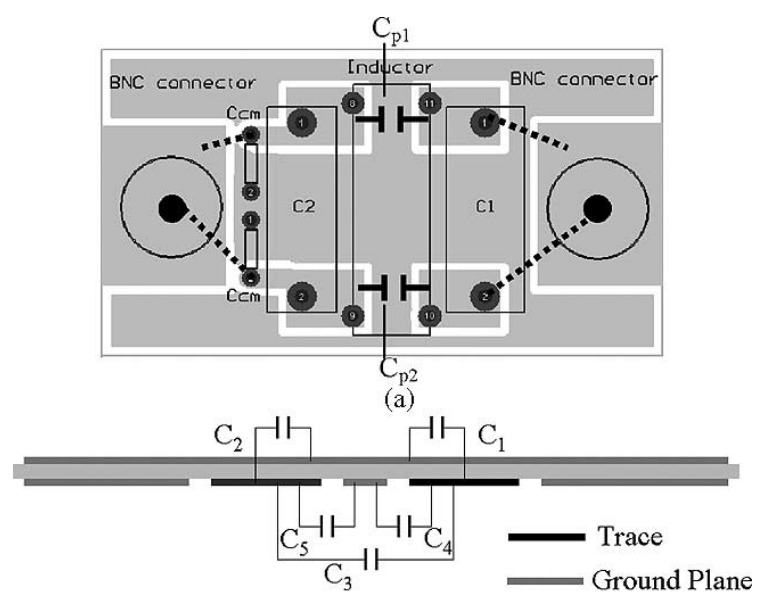

(b)

Fig. 13. Capacitive couplings between the in and out traces: (a) Equivalent capacitors on the bottom side. (b) Cross section view of the capacitive couplings.

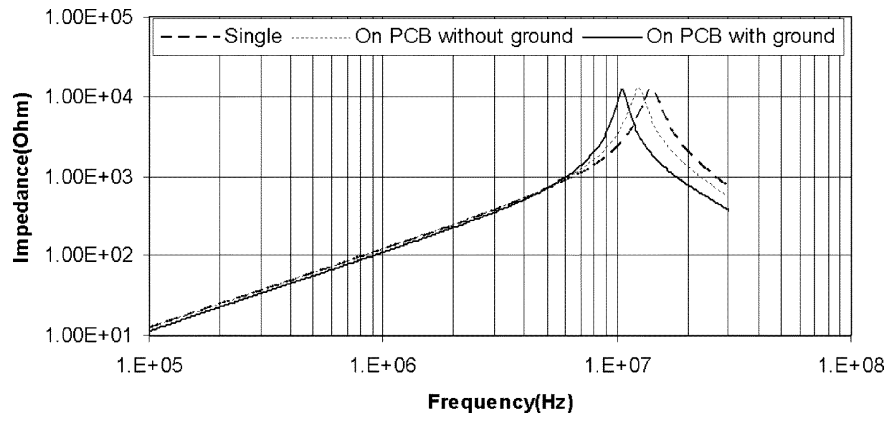

Fig. 14. Comparison of the measured inductor impedances.

TABLE II

EXTRACTED INDUCTANCE AND PARASITIC CAPACITANCE

\begin{tabular}{l|c|c|c}
\hline & $\begin{array}{c}\text { Single } \\
\text { inductor }\end{array}$ & $\begin{array}{c}\text { Mounted on the PCB } \\
\text { without the ground plane }\end{array}$ & $\begin{array}{c}\text { Mounted on the PCB } \\
\text { with the ground plane }\end{array}$ \\
\hline Resonant frequency & $14.14 \mathrm{MHz}$ & $12.4 \mathrm{MHz}$ & $10.5 \mathrm{MHz}$ \\
\hline Inductance & $18.91 \mu \mathrm{H}$ & $18.91 \mu \mathrm{H}$ & $18.91 \mu \mathrm{H}$ \\
\hline Parasitic capacitance & $6.7 \mathrm{pF}$ & $8.7 \mathrm{pF}$ & $12.5 \mathrm{pF}$ \\
\hline
\end{tabular}

mounted on a PCB without the ground plane; and in the last case, the inductor is mounted on a PCB with the ground plane. The measured impedances of these three cases are shown in Fig. 14. In Fig. 14, for the single inductor case, the impedance peak due to the parallel resonance caused by the inductance and the parasitic capacitance of the inductor is at $14.14 \mathrm{MHz}$. When the inductor is mounted on the PCB without the ground plane, the resonant frequency decreases to $12.4 \mathrm{MHz}$. It further decreases to $10.5 \mathrm{MHz}$ when the inductor is mounted on the PCB with the ground plane. The equivalent circuit parameters of the inductor can be easily derived from these impedance curves. The results are shown in Table II. From these analysis and experiments, the capacitive coupling between in and out traces is equivalent to a capacitor $C_{p}$ with a capacitance $5.8 \mathrm{pF}$ paralleled with the inductor. In this $5.8 \mathrm{pF}$ capacitance, $2 \mathrm{pF}$ is attributed to the direct capacitive coupling between in and out traces and $3.8 \mathrm{pF}$ is attributed to the capacitive coupling through the ground plane. The inductance of the inductor is reduced by $0.81 \mu \mathrm{H}$; so $M_{7}$ is $0.81 \mu \mathrm{H}$. 

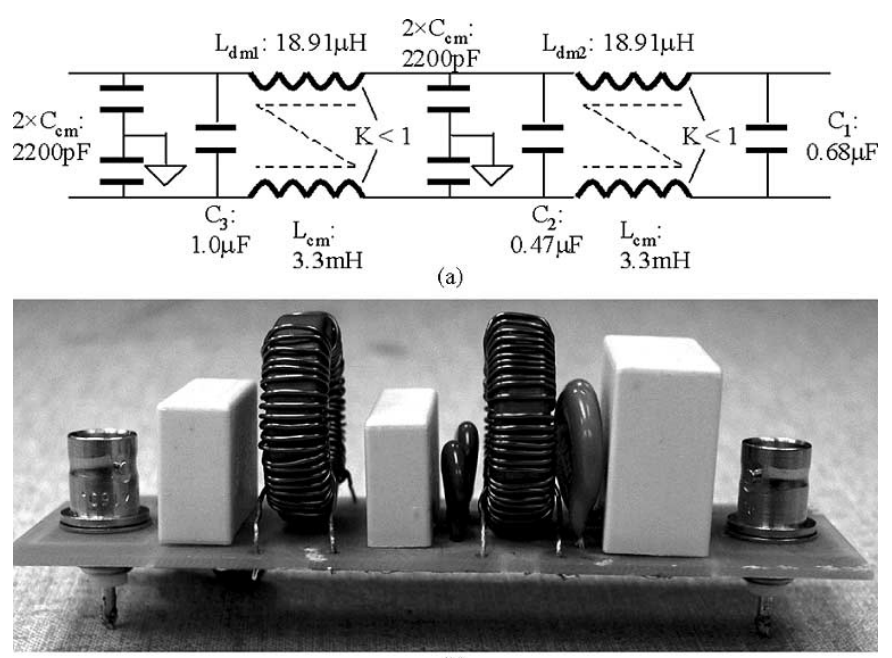

(b)

Fig. 15. Investigated $\Gamma+\Pi$ filter: (a) circuit of the filter and (b) prototye of the filter.

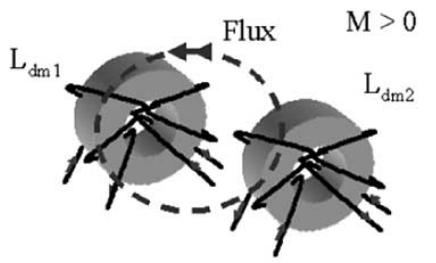

Same winding directions

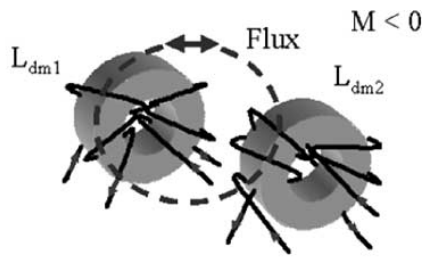

Opposite winding directions
Fig. 16. Inductive couplings between two inductors.

\section{Inductive Coupling Between In and Out Trace Loops $\left(M_{6}\right.$ in Fig. 3)}

Due to the large current difference between the in and out trace loops, the inductive coupling $M_{6}$ is also considered and extracted using S-parameters. It is around $0.2 \mathrm{nH}$. The couplings between the trace loops and capacitors are ignored and their effects are included in $M_{6}$.

\section{E. Inductive Coupling Between Two Inductors in a Two-Stage Filter}

A $\Gamma+\Pi$ filter, shown in Fig. 15, is investigated last. Because of the high permeability of inductor cores, the inductive coupling between two inductors is significant. The mutual inductance between two inductors is usually much larger than other inductive couplings on the capacitor branch, therefore the $C_{2}$ branch in Fig. 15 would be significantly affected by this coupling. The winding directions of the two inductors also affect the mutual inductance polarities, as illustrated in Fig. 16.

The mutual inductance between two inductors is extracted using S-parameters. It is $1.79 \mu \mathrm{H}$, which is much larger than the ESL $(14 \mathrm{nH})$ of the capacitors and other mutual inductances. Therefore, for $C_{2}$ branch, only the effect of the inductive coupling between two inductors is considered. Experiments show this coupling affects the filter performance much. The equivalent circuits for $C_{2}$ and two inductors are shown in Fig. 17. The resoant frequency of a $0.47 \mu \mathrm{F}$ capacitor is around $2 \mathrm{MHz}$. In
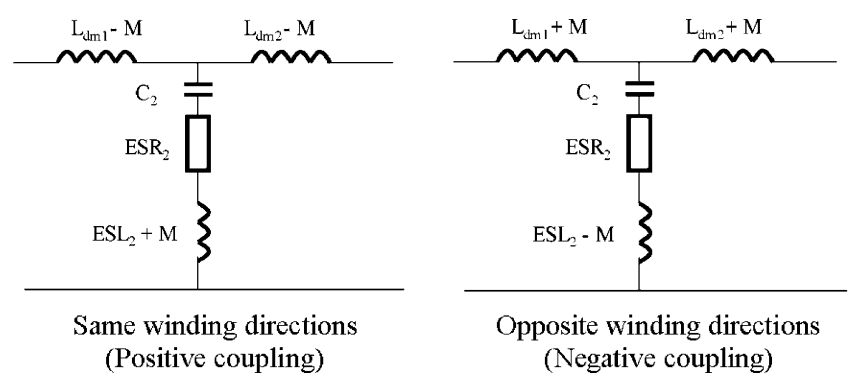

Fig. 17. Equivalent circuits for two inductors and $C_{2}$.

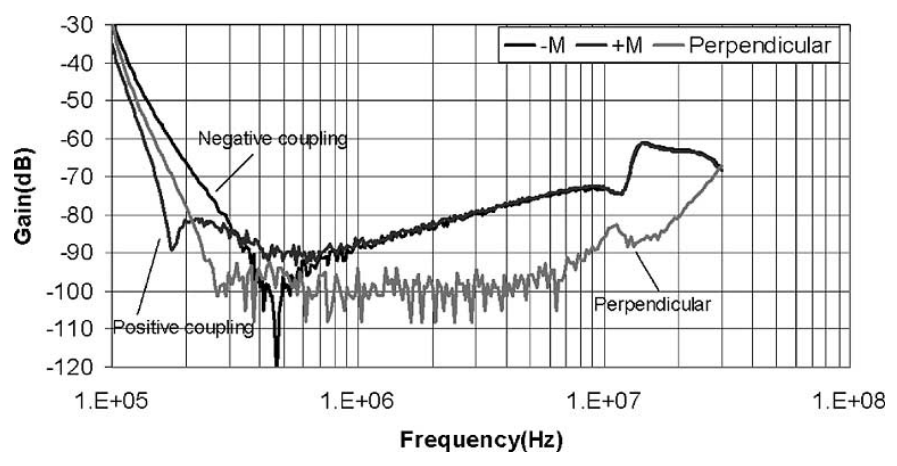

Fig. 18. Transfer-gain comparison.

Fig. 17, for the positive coupling case, the reosnant frequency of the capacitor branch would be $174 \mathrm{kHz}$ because of the $1.79 \mu \mathrm{H}$ mutual inductance. For the negative coupling case, from (5), the minimum impedance also occurs at around $174 \mathrm{kHz}$ because the mutual inductance is much larger than the ESL. It is obvious that the performance of the capacitor is strongly affected by this mutual inductance.

In order to reduce this effect, the mutual inductance should be made to be as small as possible. It is well known that two perpendicular inductors are beneficial for reducing the inductive coupling, but at the expense of larger space. Experiments are carried out for three cases. In the first case, the two inductors have the same winding direction. In the second case, the two inductors have different winding directions. In the third case, the two inductors are perpendicular. The measured transfer gains are shown in Fig. 18. In Fig. 18, for the case of positive coupling, a dip shows up at $174 \mathrm{kHz}$, which is just as predicted in Fig. 17. As a result, it has better performance in the LF range. Negative coupling is better from $330 \mathrm{kHz}$ to $1 \mathrm{MHz}$, but is almost the same as positive coupling in the HF range. The transfer gain of the perpendicular inductors gives the best performance from $200 \mathrm{kHz}$ to $30 \mathrm{MHz}$. The noise at the bottom of the curve is the background noise of the analyzer.

\section{Simulation Verification, Discussion AND RECOMMENDATIONS}

\section{A. Simulation Verification on the Extracted Models}

Since all the parasitic parameters in Fig. 3 are extracted, it is possible to simulate the transfer gain of the whole filter and then compare this with the measurement result. The equivalent 


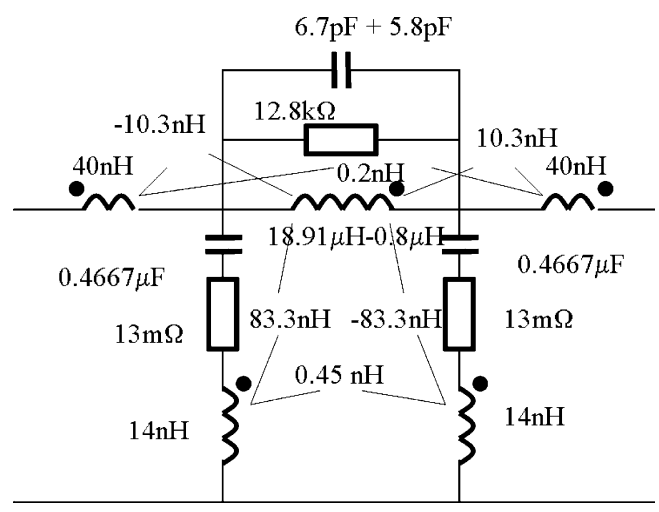

Fig. 19. EMI filter model for positive coupling.

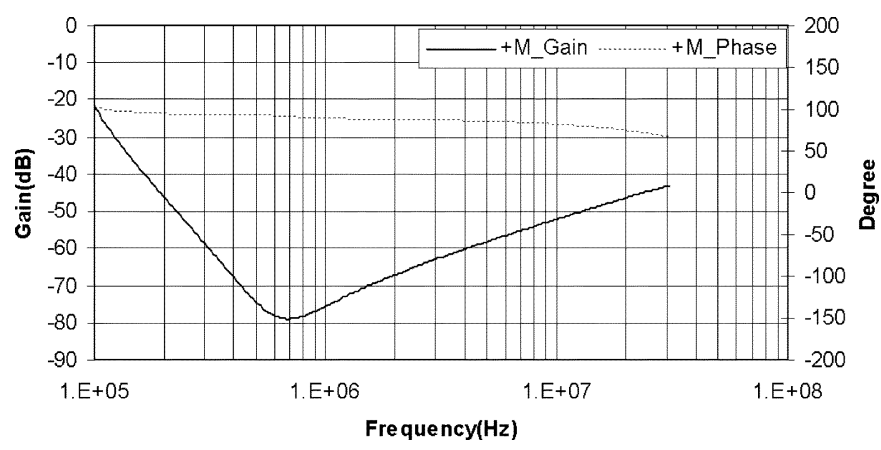

Fig. 20. Simulated transfer gain for positive coupling.

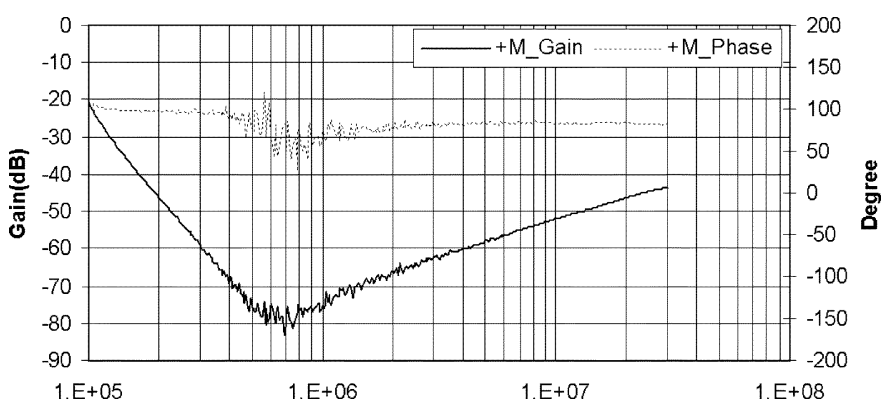

Fig. 21. Measured transfer gain for positive coupling.

circuit used for the positive coupling case is shown in Fig. 19. The coupling is defined as positive coupling because the total equivalent inductance caused by the couplings of the inductor on the capacitor branch is positive. Fig. 20 illustrates simulation result in terms of both amplitude and phase. Fig. 21 shows the measured transfer gain in terms of both amplitude and phase. From Figs. 20 and 21, they closely match.

For the negative coupling and proposed winding structures, the simulated transfer gains also match the measured transfer gains very closely. From these comparisons, the parasitic couplings are successfully characterized and extracted.

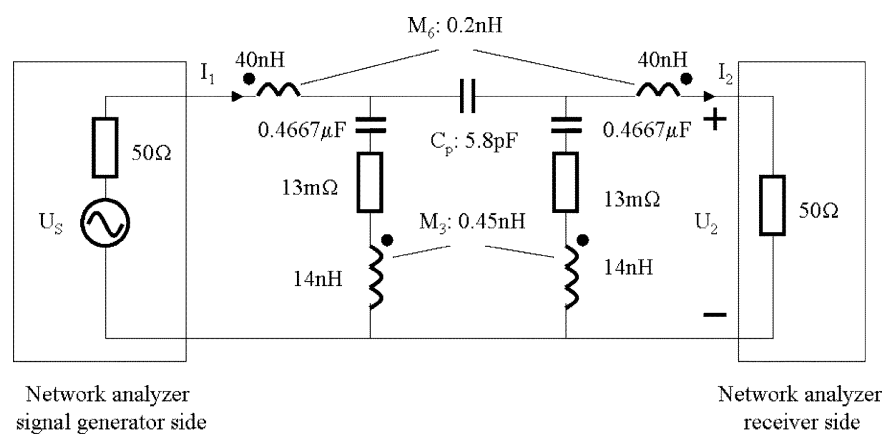

Fig. 22. Equivalent circuit when the inductor is disconnected.

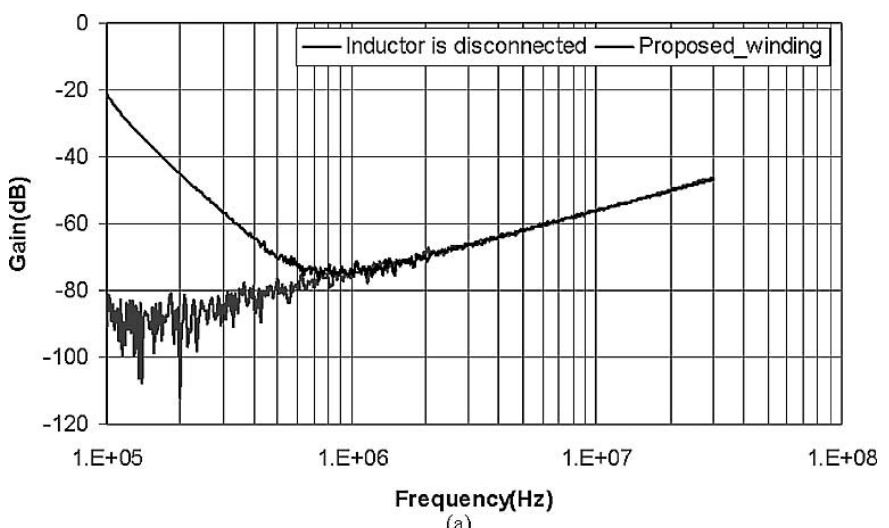

(a)

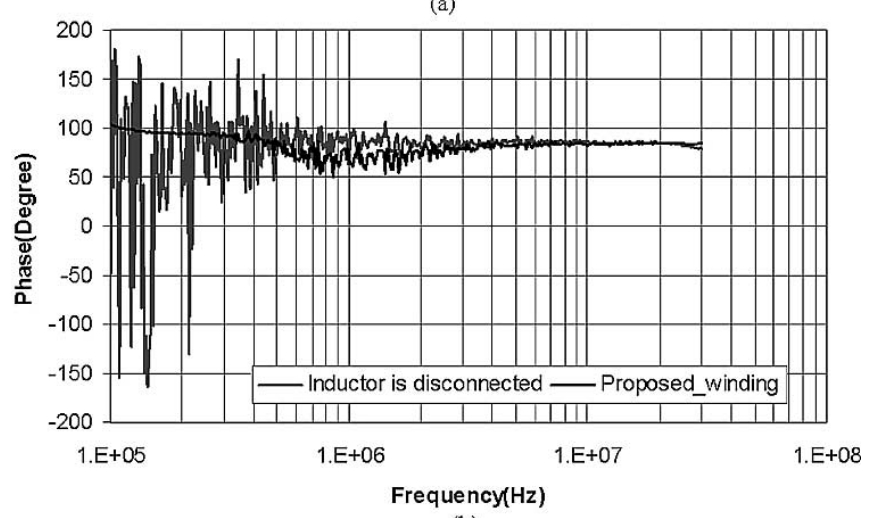

(b)

Fig. 23. Transfer-gain comparison: (a) transfer gain and (b) phase. (The noise below $500 \mathrm{kHz}$ in both diagrams is the background noise of the network analyzer).

\section{B. Discussion}

From Table I and Fig. 9, $M_{1}, M_{2}$ and $M_{4}, M_{5}$ are greatly reduced to improve the performance of the EMI filter if the proposed winding arrangement is used. In order to investigate the impact of other couplings on EMI filter performance when $M_{1}$, $M_{2}$ and $M_{4}, M_{5}$ are negligible, an experiment is carried out. In the experiment, the inductor is disconnected from the filter (but still kept on the PCB because the inductor affects the inductive couplings in the filter) and then the transfer gain is measured. The equivalent circuit for the measurement setup is shown in Fig. 22. It is obvious that the measured transfer gain is determined by $M_{3}, M_{6}$ and $C_{p}$ because the inductor is not connected to the filter. In Fig. 23, the transfer gain of the whole filter when 
the proposed winding arrangement is used is compared with this measured transfer gain.

In Fig. 23, two transfer-gain curves are almost same above 1 $\mathrm{MHz}$ no matter in gain and phase. It indicates that noise does not come through the inductor in HF range. This measurement also indicated the effect of $C_{p}$ (the capacitive coupling) is insignificant due to the small impedances of filter capacitors, which leads to a $+90^{\circ}$ phase.

In Fig. 22, $I_{1}$ is given as

$$
\dot{I}_{1} \approx \frac{\dot{U}_{S}}{50}
$$

The measured transfer gain $T_{G}$ in Fig. 22 is then described as

$$
T_{G}=\frac{2 \dot{U}_{2}}{\dot{U}_{S}} \approx \frac{j \omega}{25}\left(M_{3}+M_{6}\right)
$$

Equation (11) means because the effects of $M_{1}, M_{2}$ and $M_{4}$, $M_{5}$ are negligible, for this EMI filter the HF performance is determined by $M_{3}$ and $M_{6}$ only. If $M_{3}$ is larger than $M_{6}$, it would be the one dominates the transfer gain in HF range. That is what Fig. 12 shows.

\section{Measures Used to Improve Filter Performance}

Based on the preceding analysis and experiments, $M_{1}, M_{2}$ and $M_{3}$ are the couplings with the capacitors, so the capacitors are the components critical to the achievement of high performance. Steps can be taken to reduce these couplings.

1) Two capacitors should be kept far away enough to reduce the inductive coupling between them.

2) The capacitors and inductor should also be kept far away enough to reduce the inductive coupling between them.

3) The proposed winding arrangement can be used for the inductor to reduce the inductive coupling with capacitors.

4) Two capacitors can be shielded.

5) The pin of the capacitor should be kept as short as possible to reduce inductive couplings.

6) For a two-stage filter, two inductors can be placed in perpendicular fashion to reduce the related inductive coupling.

7) Purposely selecting the appropriate winding directions to get either positive or negative mutual inductance.

$M_{4}, M_{5}$, and $M_{6}$ are the couplings with the in and out trace loops, so the trace loops are also important components. Steps can be taken to reduce these couplings.

1) The proposed winding arrangement can be used for the inductor to reduce the inductive coupling with trace loops.

2) The areas of in and out trace loops should be kept small to reduce the $M_{4}, M_{5}$ and $M_{6}$.

$M_{7}$ and $C_{p}$ result from the ground plane. Some steps can be taken to reduce them.

1) Do not use ground plane under the inductor to reduce $M_{7}$.
2) Keep the enough clear distance between ground plane and traces, in and out traces to reduce the direct capacitive couplings and those through the ground plane.

\section{CONCLUSION}

In this paper, six important inductive and capacitive couplings existing in the EMI filters are identified and quantified by both the experiments and the simulations. The theories on the parasitic couplings were developed and many measures are advised to reduce these couplings. It is found that the capacitors, the trace loops and the ground plane are critical to the achievement of high performance. The performance of a capacitor in an EMI filter is determined by the inductive couplings with other capacitors and inductors. The inductive couplings with the in and out trace loops also play an important role on the filter performance. The ground plane can reduce the inductance of inductors due to the eddy current and it also offers an easy path for the capacitive couplings between the in and out traces. For a two-stage filter, the inductive coupling between two inductors strongly affects the performance of the filter in the whole frequency range [1], [3]-[9].

\section{REFERENCES}

[1] S. Wang, F. C. Lee, and W. G. Odendaal, "Improving the performance of boost PFC EMI filters," in Proc. 18th Annual IEEE Applied Power Electronics Conference and Exposition (APEC'03), vol. 1, pp. 368-374.

[2] M. J. Nave, Power Line Filter Design for Switched-Mode Power Supply. New York: Van Nostrand Reinhild, 1991.

[3] H. W. Ott, Noise Reduction Techniques in Electronic Systems, Second ed. New York: Wiley, 1988.

[4] S. Wang, F. C. Lee, D. Y. Chen, and W. G. Odendaal, "Effects of parasitic parameters on EMI filter performance," in Proc. CPES Seminar, 2003.

[5] D. H. Liu and J. G. Jiang, "High frequency characteristic analysis of emi filter in switch mode power supply (smps)," in Proc. IEEE 33rd Annual Power Electronics Specialists Conference (PESC'02), vol. 4, 2002, pp. 2039-2043.

[6] Ozenbaugh and R. Lee, EMI Filter Design. New York: Marcel Dekker Inc., 1996.

[7] N. Balabanian and T. Bickart, Linear Network Theory: Analysis, Properties, Design and Synthesis. New York: Matrix Publishers, Inc., 1981.

[8] “Agilent AN154 S-Parameters Design Application Note," Agilent Technologies, 2000.

[9] R. Anderson, Test and Measurement Application Note 95-1 S-Parameters Techniques: Hewlett- Packard, 1997.

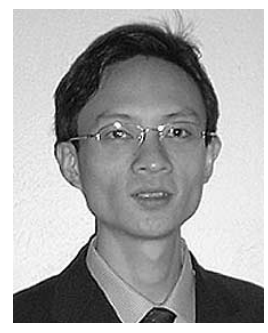

Shuo Wang (S'03) received the B.S.E.E. degree from Southwest Jiaotong University, Chengdu, China, in 1994, the M.S.E.E. degree from Zhejiang University, Hangzhou, China, in 1997, and is currently pursuing the Ph.D. degree at the Center for Power Electronics Systems (CPES), Virginia Polytechnic Institute and State University, Blacksburg.

From 1997 to 1999, he was with ZTE Telecommunication Corporation, Shenzhen, China, where he was a Senior R\&D Engineer and responsible for the development and support of the power supply for wireless products. In 2000, he worked at UTstarcom Telecommunication Corporation, Hangzhou, China, where he was responsible for the development and support of the optical access networks.

Mr. Wang received the excellent R\&D Engineer Award in 1998. 


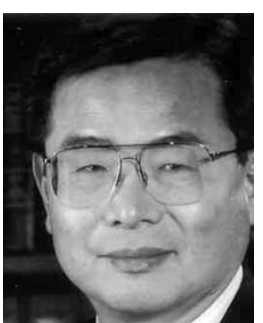

Fred C. Lee (S'72-M'74-SM'87-F'90) received the B.S. degree in electrical engineering from the National Cheng Kung University, Taiwan, R.O.C., in 1968 and the M.S. and Ph.D. degrees in electrical engineering from Duke University, Durham, NC, in 1971 and 1974, respectively.

Dr. Lee is currently a University Distinguished Professor at Virginia Tech. He is a University Distinguished Professor with Virginia Polytechnic Institute and State University (Virginia Tech), Blacksburg, and prior to that he was the Lewis A. Hester Chair of Engineering at Virginia Tech. He directs the Center for Power Electronics Systems (CPES), a National Science Foundation engineering research center whose participants include five universities and over 100 corporations. In addition to Virginia Tech, participating CPES universities are the University of Wisconsin-Madison, Rensselaer Polytechnic Institute, North Carolina A\&T State University, and the University of Puerto Rico-Mayaguez. He is also the Founder and Director of the Virginia Power Electronics Center (VPEC), one of the largest university-based power electronics research centers in the country. VPEC's Industry-University Partnership Program provides an effective mechanism for technology transfer, and an opportunity for industries to profit from VPEC's research results. VPEC's programs have been able to attract world-renowned faculty and visiting professors to Virginia Tech who, in turn, attract an excellent cadre of undergraduate and graduate students Total sponsored research funding secured by him over the last 20 years exceeds $\$ 35$ million. His research interests include high-frequency power conversion, distributed power systems, space power systems, power factor correction techniques, electronics packaging, high-frequency magnetics, device characterization, and modeling and control of converters. He holds 19 U.S patents, and has published over 120 journal articles in refereed journals and more than 300 technical papers in conference proceedings.

Dr. Lee received the Society of Automotive Engineering's Ralph R. Teeter Education Award (1985), Virginia Tech's Alumni Award for Research Excellence (1990), and its College of Engineering Dean's Award for Excellence in Research (1997), in 1989, the William E. Newell Power Electronics Award, the highest award presented by the IEEE Power Electronics Society for outstanding achievement in the power electronics discipline, the Power Conversion and Intelligent Motion Award for Leadership in Power Electronics Education (1990), the Arthur E. Fury Award for Leadership and Innovation in Advancing Power Electronic Systems Technology (1998), the IEEE Millennium Medal, and honorary professorships from Shanghai University of Technology, Shanghai Railroad and Technology Institute, Nanjing Aeronautical Institute, Zhejiang University, and Tsinghua University. $\mathrm{He}$ is an active member in the professiona community of power electronics engineers. He chaired the 1995 International Conference on Power Electronics and Drives Systems, which took place in Singapore, and co-chaired the 1994 International Power Electronics and Motion Control Conference, held in Beijing. During 1993-1994, he served as President of the IEEE Power Electronics Society and, before that, as Program Chair and then Conference Chair of IEEE-sponsored power electronics specialist conferences.

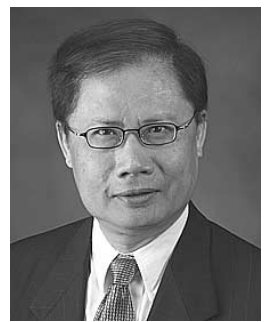

Dan Y. Chen (S'72-M'75-SM'83-F'02) received the B.S.E.E degree from National Chiao-Tung University, Taiwan, R.O.C., in 1969 and the Ph.D. degree in electrical engineering from Duke University, Durham, NC, in 1975.

From 1975 to 1979 , he worked for GE Corporate Research Center, Schenectady, NY, working on solidstate power applications. From 1979 to 2003, he was with the Electrical Engineering Department, Virginia Polytechnic Institute and State University (Virginia Tech), Blacksburg, as a Full Professor. He was a core faculty of the prestigious National Science Foundation Engineering Research Center for Power Electronics Systems at Virginia Tech. Since August, 2003, he has been a Professor in the Electrical Engineering Department, National Taiwan University, Taipei, Taiwan, R.O.C. He has published one IEEE press book, over one hundred papers, one tutorial article in the prestigious IEEE Spectrum magazine, and holds seven U.S. patents all in the field of power electronics. His research interest includes power conversion applications including electronic ballast, electric car, high power semiconductor device characterization, EMI in switching circuits, modern magnetic amplifier for switching power supplies, and power management IC architecture and design.

Dr. Chen received the IEEE Aerospace society Barry Carlton award in 1973 , the best paper award of the 1998 IEEE Power Electronics Society, and the NASA invention award in 1984

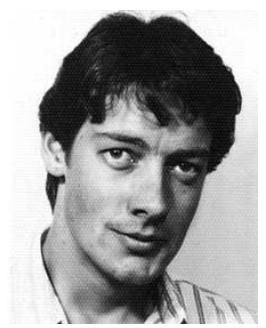

Willem Gerhardus Odendaal (M'98) was born in South Africa in 1969. He received the B.Eng., M.Eng., and D.Eng. degrees in electrical and electronics engineering from Rand Arikaans University, Johannesburg, South Africa, in 1992, 1995, and 1997, respectively.

He spent one year in a post-doctoral position under two fellowships at the Virginia Power Electronics Center, Virginia Polytechnic Institute and State University (Virginia Tech), Blacksburg, before joining Philips Research North America in New York as a Senior Member of Research Staff. Since Fall 2001, he has been Assistant Professor in the Bradley Department of Electrical and Computer Engineering, Virginia Tech, as well as a faculty member of the NSF Engineering Research Center for Power Electronics Systems (CPES). His research interests include electromagnetic and thermodynamic energy processing and packaging of power electronic circuits.

Dr. Odendaal is Chairman of the Power Electronics Devices and Components Committee of the IEEE Industry Applications Society. 